\title{
Circular Dichroism and Superdiffusive Transport at the Surface of BiTeI
}

\author{
J. Mauchain, ${ }^{1}$ Y. Ohtsubo, ${ }^{2}$ M. Hajlaoui, ${ }^{1}$ E. Papalazarou,,${ }^{1}$ M. Marsi, ${ }^{1}$ A. Taleb-Ibrahimi, ${ }^{2}$ J. Faure, ${ }^{3}$ \\ K. A. Kokh, ${ }^{4}$ O. E. Tereshchenko, ${ }^{5}$ S. V. Eremeev,${ }^{6,7}$ E. V. Chulkov, ${ }^{6,8}$ and L. Perfetti ${ }^{9}$ \\ ${ }^{1}$ Laboratoire de Physique des Solides, CNRS-UMR 8502, Université Paris-Sud, FR-91405 Orsay, France \\ ${ }^{2}$ Synchrotron SOLEIL, L'Orme des Merisiers, Saint-Aubin-BP 48, F-91192 Gif sur Yvette, France \\ ${ }^{3}$ Laboratoire d'Optique Appliquée, Ecole polytechnique, 91128 Palaiseau cedex, France \\ ${ }^{4}$ Sobolev Institute of Geology and Mineralogy, Siberian Branch, Russian Academy of Sciences, \\ Koptyuga pr. 3, Novosibirsk 630090 Russian Federation \\ ${ }^{5}$ Rzhanov Institute of Semiconductor Physics, Siberian Branch, Russian Academy of Sciences, \\ and Novosibirsk State University, Novosibirsk, 630090 Russian Federation \\ ${ }^{6}$ Tomsk State University, 634050 Tomsk, Russian Federation \\ ${ }^{7}$ Institute of Strength Physics and Materials Science SB RAN, 634021 Tomsk, Russian Federation \\ ${ }^{8}$ Donostia International Physics Center (DIPC), CFM-MPC, Centro Mixto CSIC-UPV/EHU, \\ and Departamento de Física de Materiales, UPV/EHU, 20018 San Sebastián, Spain \\ ${ }^{9}$ Laboratoire des Solides Irradiés, Ecole polytechnique, 91128 Palaiseau cedex, France
}

(Received 21 May 2013; published 19 September 2013)

\begin{abstract}
We investigate the electronic states of BiTeI after the optical pumping with circularly polarized photons. Our data show that photoexcited electrons reach an internal thermalization within 300 fs of the arrival of the pump pulse. Instead, the dichroic contrast generated by the circularly polarized light relaxes on a time scale shorter than $80 \mathrm{fs}$. This result implies that orbital and spin polarization created by the circular pump pulse rapidly decays via manybody interaction. The persistent dichroism at longer delay times is due to the helicity dependence of superdiffussive transport. We ascribe it to the lack of inversion symmetry in an electronic system far from equilibrium conditions.
\end{abstract}

DOI: 10.1103/PhysRevLett.111.126603

The application of surface and interface states in spintronic devices motivates an increasing interest in semiconductors with sizable spin-orbit interaction. The ternary compound BiTeI is a valuable example of a noncentrosymmetric material with surface states supporting large spin polarization [1-5]. A macroscopic spin injection in these surface states may be obtained by optically pumping the system with circularly polarized photons. Such a technique has been widely employed to optically orient the spins of bulk semiconductors [6] and GaAs/AlAs quantum wells [7]. Here the optical transition selection rules of the Bloch bands are inherited from their parent atomic orbits. Alternatively, the spin polarization can be entangled to valley degrees of freedom by intercellular currents [8]. It has been shown in monolayer $\mathrm{MoS}_{2}$ that this spin polarization comes along with the contrasted circular dichroism in different regions of the Brillouin zone $[9,10]$. As for the aforementioned systems, BiTeI also holds a large spinorbit coupling and lacks inversion symmetry. These properties lead to a finite spin orientation upon photoexcitation with circular polarized beams [11]. In spite of this, the surface of BiTeI does not have a charge gap but metallic electronic states $[1,3,4]$. Therefore, fast scattering channels can relax the orbital and spin polarization on a femtosecond time scale. On the other hand, superdiffusive transport from the surface towards the bulk leaves a persistent trace of the initial polarization for a longer time.

This Letter aims for a better understanding of photoexcited states and the electronic transport phenomena at
PACS numbers: 72.25.Fe, 72.25.Ba, 73.20.At

the surface of materials with strong spin-orbit interaction and without inversion symmetry. The ternary compound BiTeI is a benchmark example in which to investigate the electronic evolution after an optical perturbation. We probe the electronic states in the transient regime by means of time resolved photoelectron spectroscopy. Our experimental technique has the unique capability of detecting the population of electronic states upon photoexcitation by pump pulses with different helicities. By these means, we observed an internal thermalization of the photoexcited electrons within $300 \mathrm{fs}$ of the arrival of the pump pulse. Interestingly, the dichroic contrast acquired $80 \mathrm{fs}$ after photoexcitation is already different from the one expected from the point group symmetry of the crystal. We deduce that the initial orbital and spin orientation decay on a time scale much faster than the electronic thermalization. The persistent dichroic contrast arises from the helicity dependence of the superdiffusive transport of the electrons that are highly out of equilibrium. This transport phenomenon occurs only in the period when nonthermalized electrons do not respect the detailed balanced conditions and it is strictly linked to photogalvanic effects [12].

Photoelectron spectra with a photon energy of $28 \mathrm{eV}$ have been collected at the Cassiopée beam line of the Soleil Synchrotron. Time resolved photoelectron spectroscopy experiments have been instead performed with the FemtoARPES setup, using a Ti:sapphire laser that generates $35 \mathrm{fs}$ pulses centered at $790 \mathrm{~nm}$ with a repetition rate of $250 \mathrm{kHz}$. Part of the beam is employed to generate the 
fourth harmonic by a cascade of frequency mixing in BBO crystals $\left(\beta-\mathrm{BaB}_{2} \mathrm{O}_{4}\right)$ [13]. The $197.5 \mathrm{~nm}$ probe and the $790 \mathrm{~nm}$ pump are focused on the sample with a spot diameter of 100 and $200 \mu \mathrm{m}$, respectively. Their cross correlation in a BBO crystal has a full width at half maximum of $80 \mathrm{fs}$. The bandwidth of the $197.5 \mathrm{~nm}$ beam $(6.3 \mathrm{eV})$ limits the overall energy resolution of TRPES spectra to $60 \mathrm{meV}$. All the time resolved measurements have been performed at the base temperature of $130 \mathrm{~K}$ and base pressure of $10^{-10}$ mbar. The samples are high quality single crystals of BiTeI cleaved in situ just before the data acquisition. If not differently specified, the employed pump fluence is $30 \mu \mathrm{J} / \mathrm{cm}^{2}$. The crystal growth was done by the modified Bridgman method with a rotating heat field [14]. For structural optimization and electronic band calculations, we employed the VASP code [VASP, PAW] (computational details are given in Refs. [2,5]) with the generalized gradient approximation [15] for the exchange-correlation potential. The Hamiltonian contained scalar relativistic corrections, and the SOI was taken into account by the second variation method [16].

As shown by Fig. 1(a) BiTeI is a polar compound in which $\mathrm{Bi}, \mathrm{Te}$, and I form stacking layers with the structure of a honeycomb lattice. The system admits a threefold rotation around the $c$ axis and lacks inversion symmetry. Due to the large spin-orbit interaction and small band gap the electronic states hold a large Rasbha-like splitting [5,17]. The strong band bending at the BiTeI-vacuum interface induces the Fermi level crossing of bulklike states as well as the formation of surface states. Spin resolved ARPES measurements of the surface states report an inplane polarization with helical spin texture $[1,4]$. Bulk bands hold a similar structure but generate closed Fermi surfaces around the $A$ symmetry point of the $3 \mathrm{D}$ Brillouin
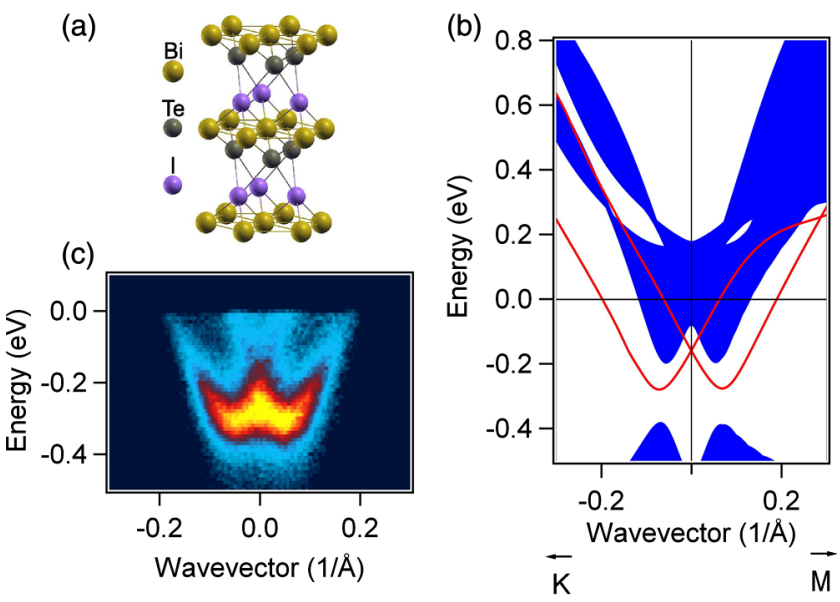

FIG. 1 (color online). (a) Crystal structure of BiTeI. (b) Calculated band structure of the Te terminated BiTeI surface. The surface states (red lines) display large spin-orbit splitting and partially overlap with the projected bulk bands (blue area). (c) Photoelectron intensity map acquired along the $\Gamma-K$ direction with a photon energy of $28 \mathrm{eV}$. zone [4]. Although the cleavage of BiTeI may expose different terminations [3], our data are indicative of a surface with topmost Te atoms and downward band bending. We show in Fig. 1(b), the calculated band structure of the Te terminated surface. The two-dimensional electronic pockets generated by the surface states extend to higher binding energy and partially overlap with the bulk states. Figure 1(c) displays a photoelectron intensity map acquired along the $\Gamma-K$ direction with a photon energy of $28 \mathrm{eV}$. The two dispersing branches are surface states split by the spin-orbit interaction. The bulk electronic states are not visible at this photon energy because of the extreme surface sensitivity of the photoemission spectroscopy. Instead, the surface and bulk states attain comparable photoemission intensity at a photon energy below $7 \mathrm{eV}$ [1]. The intensity map of Fig. 2(a) has been obtained with photons of $6.3 \mathrm{eV}$ and it is compatible with previous ARPES experiments based on laser sources [1]. Even if individual bands cannot be discriminated, the size and shape of the electronic pockets match well the calculations of Fig. 1(b) (see also the Supplemental Material [18]).

Next, we focus on the dynamics of the electrons in nonequilibrium conditions. Figures 2(b)-2(d) shows
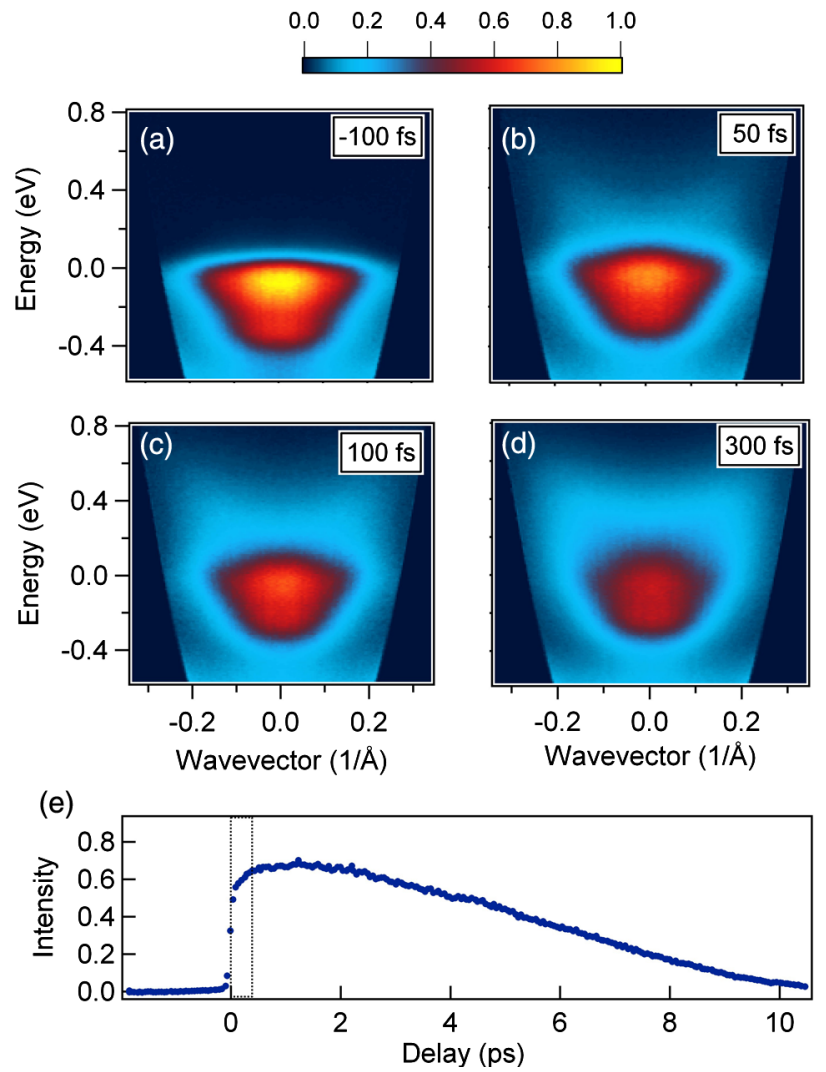

FIG. 2 (color online). (a)-(d) Photoelectron intensity maps acquired along the $\Gamma$ - $K$ direction for different pump probe delays (photon energy of $6.3 \mathrm{eV}$ ). (e) Photoelectron intensity at $0.15 \mathrm{eV}$ above the Fermi level as a function of pump-probe delay. The dashed lines indicate the temporal window of the electronic thermalization. 
intensity maps at different delay times after the arrival of the linearly polarized pump beam. Upon photoexcitation, the electronic states above the Fermi level become partially filled. The largest part of the primary excitations takes place in bulklike states, populating two dispersing states up to $0.8 \mathrm{eV}$ [see Fig. 2(b)]. The temporal evolution reported by the intensity maps of Figs. 2(a)-2(d) indicates that the Fermi liquid thermalizes by electron-electron interaction in $\cong 300 \mathrm{fs}$ (see also the movie in the Supplemental Material [18]). During this quasiadiabatic transient, the impact ionization channels increase the spectral intensity in electronic states near the Fermi level. Afterwards, the hot electrons can be described by a Fermi-Dirac function with an effective temperature and a chemical potential (for the EDCs analysis see the Supplemental Material [18]). We trace the temporal evolution of BiTeI by monitoring the photoelectron intensity at an energy $0.15 \mathrm{eV}$ above the Fermi level [see Fig. 2(e)]. This signal displays a steplike increase followed by a subpicosecond rise and a steady reduction. The cooling of hot electrons by phonon emission lasts $\sim 10 \mathrm{ps,} \mathrm{until}$ local equilibrium is established. Notice that similar time scales have been observed also in elemental bismuth [19] and topological insulators [20].

Next, we describe the dichroic properties of BiTeI both in equilibrium and after photoexcitation. Figure 3(a) shows the geometry of the FemtoARPES setup for the dichroic measurements. The electrons are always detected in the incidence plane of the incoming light (namely the $x y$ plane). Both the pump and probe beams define an angle of $45^{\circ}$ with respect to the surface normal. We are going to compare the results of two complementary experiments: (i) circular-probe measurements monitor the dichroic contrast obtained by switching the circular polarization of the probe photons and damping the pump beam and (ii) circular-pump measurements provide instead the dichroic contrast collected by switching the circular polarization of the pump-beam and polarizing the probe beam in the $x y$ plane. Before any measurement, we have verified that the intensities of different polarizations are equal within an uncertainty of $1 \%$.

Figure 3(b) shows the circular-probe signal along the $\Gamma-K$ direction as a function of energy and electronic wave vector. The contrast is maximal for electronic states lying $\sim 0.1 \mathrm{eV}$ below the Fermi level and has a nearly odd parity with respect to the in-plane wave vector. We disclose the symmetry of this signal by integrating the photoelectron intensity in the $[-0.2,0] \mathrm{eV}$ interval and varying the orientation of the sample with respect to the $x y$ plane. As shown in Fig. 3(c), the resulting contrast changes sign on the $\Gamma-M$ direction and is maximal along the $\Gamma-K$ direction. The observed dichroism is a very general property of $C_{3 v}$ surfaces, arising from the inherent lack of the inversion symmetry in a photoemission process [21]. When the electronic states hold large spin-orbit splitting, the

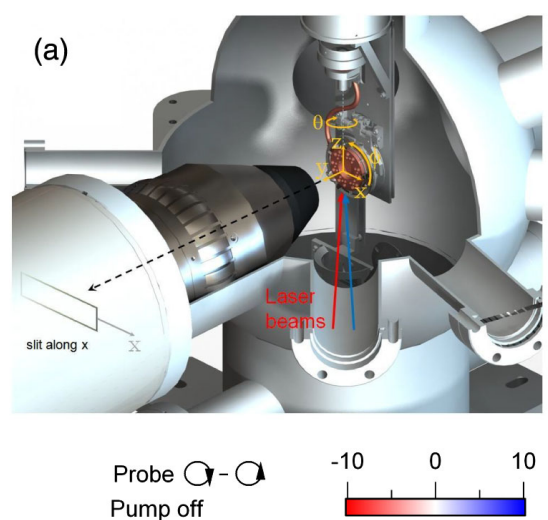

(c)
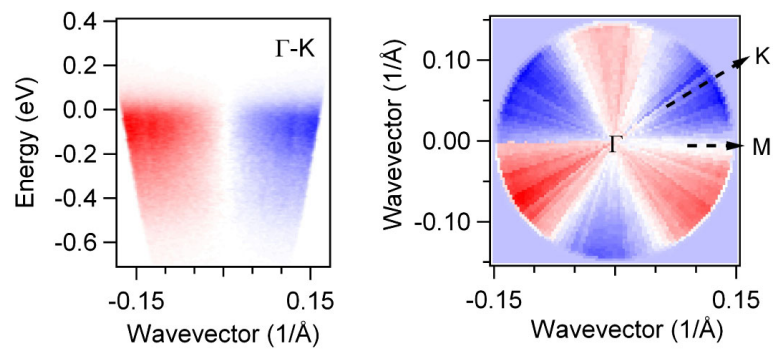

FIG. 3 (color online). (a) Experimental geometry of our experimental setup. The electrons are detected in the incident plane of the laser beams ( $x y$ plane). Both the pump and probe beams define an angle of $45^{\circ}$ with respect to the surface normal. (b) Dichroic map generated by switching the circular polarization of the probe beam and integrating the electronic states in an acceptance window of $0.2 \mathrm{eV}$ centered at $-0.1 \mathrm{eV}$. (c) Map of dichroic contrast generated by the probe beam along the $\Gamma-K$ direction. These data have been acquired without the pump beam. The scale of the dichroic contrast is percent of the average signal (left + right $) / 2$.

circular-probe dichroism can be also linked to the spin polarization [22]. As a consequence, dichroic maps have been readily employed to gain information about spin textures in topological insulators [23-25]. In our case, this comparison could be compelling only if the individual bands were clearly resolved. Nonetheless, the general trend in Fig. 3(c) may be compatible with an out-of-plane buckling of the spins in the electronic pockets $[22,26]$.

Figure 4(a) shows a circular-pump map acquired in the $\Gamma-K$ direction at a delay time of $80 \mathrm{fs}$. An almost identical map has been measured along the $\Gamma-M$ direction or other directions of the surface plane. The dichroic signal of Fig. 4(a) is nearly even with respect to $k_{\|}$and changes sign at the Fermi level. This result strongly deviates from the scenario expected in an independent particle picture. Indeed, the atomic structure of the BiTeI surface has strong similarity to a honeycomb lattice without inversion symmetry. If manybody interactions could be dismissed, the absorption difference between left- and right-handed light would display a strong wave vector dependence. In particular, the dichroic signal should be maximal along the $\Gamma-K$ direction and with nearly-odd parity with respect to $k_{\|}$[9]. Based on these general considerations, we conclude 


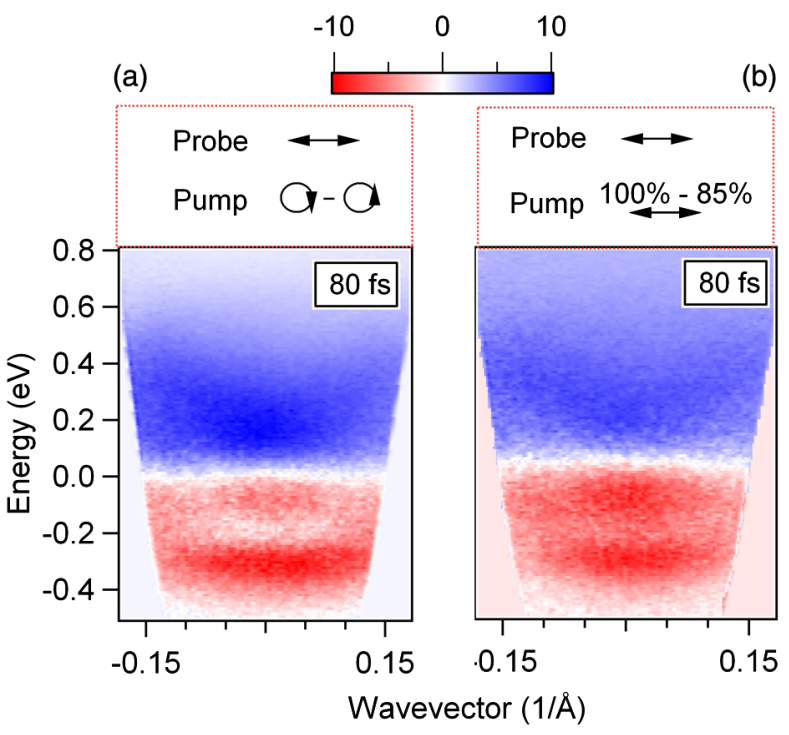

FIG. 4 (color online). (a) Map of the dichroic contrast acquired at a delay time of $80 \mathrm{fs}$ and generated by switching the circular polarization of the pump beam. The scale of the dichroic contrast is percent of the average signal (left + right)/2. (b) Difference between the photoelectron intensity maps acquired with pump fluence of $30 \mu \mathrm{J} / \mathrm{cm}^{2}$ (reference fluence) and $25 \mu \mathrm{J} / \mathrm{cm}^{2}$ ( $85 \%$ of the reference fluence) at a delay time of $80 \mathrm{fs}$.

that the circular-pump map of Fig. 4(a) should look similar to the circular-probe map of Fig. 3(b). In this case the photoexcited system would also acquire a macroscopic spin and orbital polarization $[7,8,11]$. Heuristic arguments suggest that the spin polarization should be roughly given by the ratio between the average spin-orbit splitting $\Delta_{\text {SO }}$ and the pump photon energy $\hbar \omega$ [11]. By estimating $\Delta_{\text {SO }} \sim 0.2 \mathrm{eV}$ and knowing $\hbar \omega=1.56 \mathrm{eV}$, the resulting polarization should be $\Delta_{\text {So }} / \hbar \omega \sim 10 \%$. Clearly, the presence of interactions inevitably relaxes the population imbalance generated upon the absorption of circularly polarized light. Nonetheless, a nearly odd circular-pump map could still be expected at early delay times. An interesting example has been reported in monolayer molybdenum disulphide, where the photoexcited carriers get trapped in valleys at the $K$-point of the Brilloiun zone. Apparently, the intervalley scattering of monolayer $\mathrm{MoS}_{2}$ is so weak that the circular-pump dichroism leads to a sizable polarization of the photoluminescence $[9,10]$. The reason for such weak intervalley scattering is the formation of excitons in the spin-polarized valleys [10]. As a consequence electron-hole pairs that would scatter from the $K$ to $K^{\prime}$ valley have to overcome an energy barrier that roughly corresponds to the ionization potential of the exciton. A different instance takes place in BiTeI, since no energy gaps and valleys can stabilize excitons. In contrast, the photoexcited electrons thermalize within $\cong 300$ fs near the zone center. In principle, a detectable orbital and spin polarization may still be expected during this thermalization time. However, the circular-pump map of Fig. 4(a) indicates that the measured dichroism attains an evenlike symmetry already at delay times of 80 fs. This finding suggests that manybody scattering erases the orbital and spin polarization within the duration of the pump pulse. The persistent dichroic signal of $\cong 15 \%$ is the long living trace of the helicity dependent excitation set in by the pump pulse. The measured contrast changes sign at the Fermi level, therefore mimicking the difference between two electronic distributions with different electronic temperature. This analogy is corroborated by Fig. 4(b), showing the difference between the intensity maps acquired with a pump fluence of $30 \mu \mathrm{J} / \mathrm{cm}^{2}$ and $25 \mu \mathrm{J} / \mathrm{cm}^{2}$. For both photoexcitation intensities the pump polarization is linear and the pump-probe delay is 80 fs. The similarity between the dichroic map in Fig. 4(a) and the map of Fig. 4(b) confirms that at a delay time of $80 \mathrm{fs}$, the only information retained by the circular-pump signal regards the different energy density at the surface of the system. We stress that this effect cannot be ascribed to optical activity. It is not questioned that gyrotropic absorption is present in noncentrosymmetric structures with low point group symmetry [27,28]. However, the helicity dependent absorption stems from the weak spatial dispersion of the light. Its magnitude is typically lower than $0.1 \%$ and cannot explain our experiment [28]. Instead, we ascribe the $15 \%$ dichroism to the transport of electrons out of the photoexcitation volume within the duration of the pump pulse. Similar superdiffusive currents have been proposed also in the context of the ultrafast demagnetization $[29,30]$. In the case of BiTeI, the ultrafast transport depends on the photon helicity because of the lack of spatial inversion symmetry. Indeed, we have experimentally verified that no circularpump contrast can be detected in bulk centrosymmetric systems such as bismuth and $\mathrm{Bi}_{2} \mathrm{Te}_{3}$. We conclude that the different transport of energy density is due to chiral scattering events of an electronic distribution that does not respect detailed balanced conditions. In this sense, the observed dichroism shares common aspects with photogalvanic effects and circular photocurrents [12].

In conclusion, we have characterized the dynamics of electronic states of photoexcited BiTeI. The thermalization of the electronic system occurs within $300 \mathrm{fs}$ of the arrival of the pump beam. Upon excitation with circularly polarized pulses we observe a circular dichroism with a symmetry that differs from the one expected from primary optical excitations. We conclude that spin and orbital polarization relax on a time scale faster than $80 \mathrm{fs}$. The expected temporal evolution of this dichroic contrast could resemble the relaxation of transient currents in image potential states [31]. Future experiments with shorter pulse duration may be capable of capturing this dynamics. On the other hand, the persistent dichroism already indicates that the energy density at the surface of the sample depends on the helicity of the pump pulse. We ascribe this effect to superdiffusive transport occurring while the system is out 
of equilibrium. The experimental evidence of this transport phenomenon may be of interest in related subjects such as ultrafast demagnetization $[29,30]$.

We acknowledge that the FemtoARPES project was financially supported by the RTRA Triangle de la Physique, and the ANR program Chaires d'Excellence (Nr. ANR-08-CEXCEC8-011-01).

[1] K. Ishizaka et al., Nat. Mater. 10, 521 (2011).

[2] S. V. Eremeev, I. A. Nechaev, Yu. M. Koroteev, P. M. Echenique, and E. V. Chulkov, Phys. Rev. Lett. 108, 246802 (2012).

[3] A. Crepaldi et al., Phys. Rev. Lett. 109, 096803 (2012).

[4] G. Landolt et al., Phys. Rev. Lett. 109, 116403 (2012).

[5] S. V. Eremeev, I. A. Nechaev, and E. V. Chulkov, JETP Lett. 96, 437 (2012).

[6] M. I. Dyakonov and V.I. Perel, in Optical Orientation, edited by F. Meier and B.P. Zakharchenya (Elsevier, Amsterdam, 1984).

[7] S. Pfalz, R. Winkler, T. Nowitzki, D. Reuter, A. Wieck, D. Hägele, and M. Oestreich, Phys. Rev. B 71, 165305 (2005).

[8] W. Yao, Di Xiao, and Q. Niu, Phys. Rev. B 77, 235406 (2008).

[9] T. Cao et al., Nat. Commun. 3, 887 (2012).

[10] K.F. Mak, K. He, J. Shan, and T.F. Heinz, Nat. Nanotechnol. 7, 494 (2012).

[11] B. S. Mendoza and J. L. Cabellos, Phys. Rev. B 85, 165324 (2012).

[12] V. I. Belinicher and B. I. Sturman, Usp. Fiz. Nauk 130, 415 (1980).

[13] J. Faure, J. Mauchain, E. Papalazarou, W. Yan, J. Pinon, M. Marsi, and L. Perfetti, Rev. Sci. Instrum. 83, 043109 (2012).

[14] K. A. Kokh, B. G. Nenashev, A. E. Kokh, and G. Yu. Shvedenkov, J. Cryst. Growth 275, e2129 (2005).
[15] J. P. Perdew, K. Burke, and M. Ernzerhof, Phys. Rev. Lett. 77, 3865 (1996).

[16] D. D. Koelling and B. N. Harmon, J. Phys. C 10, 3107 (1977).

[17] M. S. Bahramy, R. Arita, and N. Nagaosa, Phys. Rev. B 84, 041202 (2011).

[18] See Supplemental Material at http://link.aps.org/ supplemental/10.1103/PhysRevLett.111.126603 for the analysis of energy distribution curves, a movie showing the thermalization dynamics and additional information of circular-pump maps.

[19] E. Papalazarou et al., Phys. Rev. Lett. 108, 256808 (2012).

[20] M. Hajlaoui et al., Nano Lett. 12, 3532 (2012).

[21] M. E. Simon and C. M. Varma, Phys. Rev. Lett. 89, 247003 (2002).

[22] H. Mirhosseini and J. Henk, Phys. Rev. Lett. 109, 036803 (2012).

[23] S. R. Park et al., Phys. Rev. Lett. 108, 046805 (2012).

[24] Y.H. Wang, D. Hsieh, D. Pilon, L. Fu, D. R. Gardner, Y.S. Lee, and N. Gedik, Phys. Rev. Lett. 107, 207602 (2011).

[25] D. Niesner et al., Phys. Rev. B 86, 205403 (2012).

[26] Y. Ohtsubo, J. Mauchain, J. Faure, E. Papalazarou, M. Marsi, P. Le Fèvre, F. Bertran, A. Taleb-Ibrahimi, and L. Perfetti, Phys. Rev. Lett. 109, 226404 (2012).

[27] L. E. Golub, Europhys. Lett. 98, 54005 (2012).

[28] V.M. Agranovich and V.L. Ginzburg, in Spatial Dispersion in Crystal Optics and the Theory of Excitons, edited by R.E. Marshak (Interscience, New York, 1966).

[29] M. Battiato, K. Carva, and P. M. Oppeneer, Phys. Rev. Lett. 105, 027203 (2010).

[30] A. Eschenlohr, M. Battiato, P. Maldonado, N. Pontius, T. Kachel, K. Holldack, R. Mitzner, A. Föhlisch, P. M. Oppeneer, and C. Stamm, Nat. Mater. 12, 332 (2013).

[31] J. Güdde, M. Rohleder, T. Meier, S.W. Koch, and U. Höfer, Science 318, 1287 (2007). 